\title{
Modeling of subcontinuum thermal transport across semiconductor-gas interfaces
}

\author{
Dhruv Singh, ${ }^{1, a)}$ Xiaohui Guo, ${ }^{2}$ Alina Alexeenko, ${ }^{2}$ Jayathi Y. Murthy, ${ }^{1}$ and \\ Timothy S. Fisher ${ }^{1}$ \\ ${ }^{1}$ School of Mechanical Engineering and Birck Nanotechnology Center, Purdue University, West Lafayette, \\ Indiana 47906, USA \\ ${ }^{2}$ School of Aeronautics and Astronautics, Purdue University, West Lafayette, Indiana, USA
}

(Received 2 February 2009; accepted 20 June 2009; published online 31 July 2009)

\begin{abstract}
A physically rigorous computational algorithm is developed and applied to calculate subcontinuum thermal transport in structures containing semiconductor-gas interfaces. The solution is based on a finite volume discretization of the Boltzmann equation for gas molecules (in the gas phase) and phonons (in the semiconductor). A partial equilibrium is assumed between gas molecules and phonons at the interface of the two media, and the degree of this equilibrium is determined by the accommodation coefficients of gas molecules and phonons on either side of the interface. Energy balance is imposed to obtain a value of the interface temperature. The classic problem of temperature drop across a solid-gas interface is investigated with a simultaneous treatment of solid and gas phase properties for the first time. A range of transport regimes is studied, varying from ballistic phonon transport and free molecular flow to continuum heat transfer in both gas and solid. A reduced-order model is developed that captures the thermal resistance of the gas-solid interface. The formulation is then applied to the problem of combined gas-solid heat transfer in a two-dimensional nanoporous bed and the overall thermal resistance of the bed is characterized in terms of the governing parameters. These two examples exemplify the broad utility of the model in practical nanoscale heat transfer applications. (C) 2009 American Institute of Physics. [DOI: $10.1063 / 1.3181059]$
\end{abstract}

\section{INTRODUCTION}

Significant research effort in the recent years has been given to the understanding of thermal transport in solids at submicron scales in structures such as nanowires, nanobelts, and nanoparticles. These structures find widespread use in microelectronics, thermal interface materials, thermoelectrics, and in other emerging applications. ${ }^{1-8}$ Subcontinuum transport in gases has been studied comprehensively, particularly for high-altitude aerothermodynamics. ${ }^{9}$ With the advent of microelectromechanical systems (MEMS), the area of rarefied gas dynamics has attracted new attention in microchannel flow and heat transfer, ${ }^{10-13}$ squeeze film damping, ${ }^{14,15}$ heat transfer from microcantilevers to substrates, ${ }^{16-18}$ and in microthrusters, ${ }^{19}$ among other areas.

A variety of interesting and important physical problems have emerged out of these applications that strongly couple heat transfer in solids and gases. For example, heat generation in microcantilevers used in thermal sensing atomic force microscopy (AFM) occurs on the range of $1 \mu \mathrm{m}$ or less. The contact of the cantilever tip with the substrate is of the order of a few nanometers; the high resistance of the threedimensional (3D) contact leads to heat dissipation through the surrounding gas phase, leading to mesoscopic thermal transport in both the gaseous phase and the solid. Previous studies on thermal contact resistance of nanoparticles and nanowires on substrates have revealed that the gas gap conductance competes with the constriction resistance in the me-

${ }^{a)}$ Electronic mail: singh36@purdue.edu. soscopic regime, thereby offering a parallel pathway to heat dissipation. ${ }^{20-23}$ Heat carriers in solids (primarily phonons in a semiconductor) encounter interface and boundary scattering, leading to a reduction in their effective mean free path. These factors impede thermal transport through the solid contact and increase the importance of the gas pathway.

Another important phenomenon coupling gas and solid thermal transport is that of thermal creep, ${ }^{24}$ employed in MEMS pumps using thermal transpiration as the driving force. $^{25}$ These pumps exploit temperature gradient in the pump wall to drive flow; pump performance is contingent on the thermal gradient maintained across the solid. Published analyses ${ }^{26,27}$ have assumed linear temperature profiles in the solid, whereas, in reality, the solid temperature gradient must be computed by considering coupled gas-solid flow and heat transfer. The prediction of performance characteristics and the calibration of emerging microdevices require a clear understanding of the coupled thermofluid phenomena that accompany them.

Over the past decade, a variety of analytical and computational methods have been developed to predict heat transfer at the nanoscale. Thermal conductivity predictions in semiconductor thin films, nanowires, and superlattices have been performed by a variety of methods including the Boltzmann transport equation (BTE) ${ }^{28-33}$ atomistic Green's functions $(\mathrm{AGFs}),{ }^{34,35}$ and molecular dynamics (MD) simulation. ${ }^{36-39}$ When phase coherence effects are not important, BTE-based methods have proven very useful for mesoscale predictions in semiconductors. Most published BTE-based models have used a relaxation time approximation. ${ }^{28}$ Phonon dispersion 


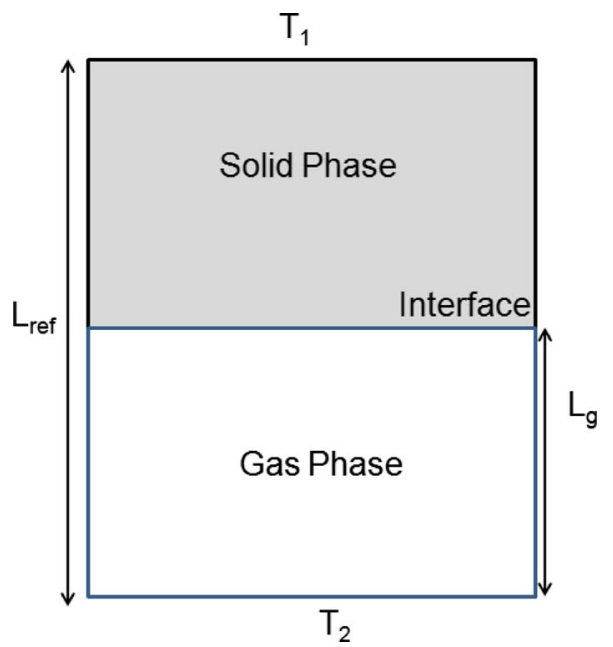

FIG. 1. (Color online) Thermal transport across a thin film-gas interface.

and scattering rates are required inputs to these models; the latter have typically been chosen to match bulk thermal conductivity data. ${ }^{33}$ Significant research has recently been directed at the direct evaluation of the phonon scattering rates incorporating the crystal structure and conservation of energy and quasimomentum of phonons. ${ }^{40,41}$ Nevertheless, models based on the relaxation time approximation remain popular because of their relative simplicity and their ability to explain experimental results. ${ }^{31,33}$

The phenomenological direct simulation Monte Carlo (DSMC) method is a widely used numerical tool for analyzing subcontinuum transport phenomena in gases. ${ }^{42}$ It can conveniently model collisions of gas molecules in free flight and the nonequilibrium between the translational, rotational, and vibrational states of gas molecules. More recently, direct solutions of the Boltzmann equation ${ }^{43-45}$ have become popular because of memory and speed advantages for very low speed flows where DSMC calculations require significant computational effort for statistically clean results.

In the present work, we develop a physically rigorous computational algorithm to simulate transport in domains that couple heat transfer in the solid and the gas. Thermal transport in the solid is assumed to be primarily by phonons. We use a finite volume solution of the Boltzmann equation under the relaxation time approximation for both the solid and the gas. For the gas, the ellipsoidal statistical BhatnagarGross-Krook (ES-BGK) model is used to calculate the velocity distribution function of gas molecules. ${ }^{46}$ For phonons, an isotropic gray assumption is made; the spatial distribution of the mean energy density of phonons of all polarizations and frequencies is simulated. ${ }^{28}$ An overall energy balance between phonons and gas molecules is imposed to determine the interface temperature. We first test the computational algorithm for the case of heat transfer between a thin film and gas, as shown in Fig. 1. A broad range of transport regimes, from free molecular flow/ballistic phonon transport to the continuum limit, is explored. Values of the interfacial thermal resistance are reported as a function of the governing nondimensional parameters. The problem of onedimensional (1D) heat transfer across the solid-gas interface serves to describe the relative effects of each governing pa- rameter on interfacial thermal resistance. Using the same computational framework, we also analyze two-dimensional (2D) heat conduction through the model nanoporous film shown in Fig. 10 to determine the importance of gas gap conduction in thermal transport across constricted solid contacts. While the results correspond to an idealized 2D constriction, the governing physics may be used to understand heat transfer mechanisms at a tip-substrate interface, in other contact geometries, and in a variety of porous beds used in emerging energy-related applications.

\section{GOVERNING EQUATIONS}

Consider an interface between a solid and a gaseous phase as shown in Fig. 1. Thermal conduction in semiconductors and dielectrics primarily occurs through lattice vibrations, the quanta of which are also referred to as phonons. Heat conduction in the solid may be described under the particle framework by the phonon BTE, neglecting effects of phonon coherence and confinement effects. For $\mathrm{Si}$, this is strictly valid for length scales greater than $5-10 \mathrm{~nm}^{47}$

A variety of phonon transport models have been developed in the literature, incorporating polarization and frequency dependence to varying degrees. ${ }^{28}$ In this paper, a gray phonon BTE under the relaxation time approximation is used to compute thermal transport in the solid. Details of polarization and frequency-dependent transmission across gas-solid interfaces are not well understood. Therefore, in this first paper, we assume that phonon properties are independent of polarization and frequency. This type of gray treatment has successfully been employed to model transport in cross-plane direction of superlattices, ${ }^{29}$ nanocomposites ${ }^{30}$ and across contacts. ${ }^{21}$

In the gas phase, gas molecules are in constant random motion and interact with each other. Transport of mass, momentum and energy in gases is described by the kinetic theory of gases. We solve for the velocity distribution function of gas molecules under the ES-BGK model for the scattering operator of the gas phase Boltzmann equation. ${ }^{43,46,48} \mathrm{~A}$ full resolution of the velocity space of gas molecules is used to describe transport. The governing equations are summarized below.

\section{A. Solid}

The Boltzmann equation for phonons represents the balance of phonon energy density in a phase element due to phonon propagation with a group velocity $v_{g}$ and a mean relaxation time $\tau$. Under the gray approximation, the steady state phonon BTE may be written as,

$$
\nabla \cdot\left(v_{g} \hat{s} e^{\prime \prime}\right)=\frac{e^{0}-e^{\prime \prime}}{\tau},
$$

where

$$
e^{\prime \prime}=\frac{1}{4 \pi} \sum_{q}\left[\int\left(f_{p}-f_{\mathrm{pref}}\right) \hbar \omega D_{q}(\omega) d \omega\right],
$$




$$
e^{0}=\frac{1}{4 \pi} \int_{4 \pi} e^{\prime \prime} d \Omega .
$$

Here, $e^{\prime \prime}(\hat{\mathbf{s}}, \mathbf{r})$ is the net energy density $\left(\mathrm{J} / \mathrm{m}^{3} \mathrm{Sr}\right)$ of all phonon groups with distribution function $f_{p}$ at position $\mathbf{r}$ and in wave-vector direction $\hat{\mathbf{s}}$ after subtracting a background energy intensity value corresponding to a phonon distribution $f_{\text {pref }}$ at $T_{\text {ref. }}$. The summation over $q$ denotes a sum over all polarizations. $e^{0}(\mathbf{r})$ represents the angular average of $e^{\prime \prime}(\hat{\mathbf{s}}, \mathbf{r})$ over all directions $\hat{\mathbf{s}}$ at a given position $\mathbf{r}$. The phonon distribution function $f_{p}$ attains the Bose-Einstein distribution at equilibrium. The gray BTE treats phonons of all polarizations and wave vectors (frequencies) as having the same group velocity $v_{g}$ and a single relaxation time $\tau$. The group velocity $v_{g}$ is chosen to reflect the velocity of the dominant phonon groups at the temperature under consideration, and $\tau$ is chosen so as to recover the bulk thermal conductivity of the solid computed as, $k_{s}=C_{p} v_{g}^{2} \tau / 3$ at the required temperature.

\section{B. Gas}

Similarly, the Boltzmann kinetic equation for gas molecules represents the balance of the number of molecules in the phase space element due to the free molecule flight and collisions. In the steady state under the BGK/ES-BGK approximation this is expressed as,

$$
\nabla .\left(c \hat{\mathbf{s}} f_{g}\right)=-\nu\left(f_{g}-f_{\gamma}\right),
$$

where $f_{g}$ is the velocity distribution function of gas molecules in the differential velocity magnitude element $(c, c$ $+d c$ ), $\nu$ is the collision frequency, $f_{\gamma}$ is an anisotropic Gaussian function used to establish local equilibrium, and is of the form

$$
f_{\gamma}=a \exp \left(-\Gamma c^{2}+\gamma_{x} c_{x}+\gamma_{y} c_{y}+\gamma_{z} c_{z}\right),
$$

where $\overrightarrow{c^{\prime}}=\left(\vec{c}-\overrightarrow{c_{0}}\right)$ is the thermal velocity of the molecules and the coefficients $a, \Gamma, \gamma_{x}, \gamma_{y}, \gamma_{z}$ must be rigorously calculated at every spatial location by enforcing conservation of mass, momentum and energy ${ }^{43}$ as,

$$
\begin{aligned}
& m \int f_{\gamma} d^{3} \vec{c}=\rho, \\
& m \int c_{i} f_{\gamma} d^{3} \vec{c}=\rho c_{0 i}, \\
& m \int \frac{c^{\prime 2}}{2} f_{\gamma} d^{3} \vec{c}=\frac{3}{2} \rho R T .
\end{aligned}
$$

However, a BGK model for the calculation of $f_{\gamma}$ does not result in physically realistic transport coefficients. In particular, the model always produces a Prandtl number of unity. $^{43,49}$ Several new models for the relaxation terms have emerged, the most common being the ES-BGK, which fits both Prandtl number and the second coefficient of viscosity in the Navier-Stokes approximation. ${ }^{46,48}$ In addition to mass and momentum conservation, the ES BGK model correctly reproduces the stress tensor. This calls for the use of more coefficients in the anisotropic Gaussian to represent a weakly nonequilibrium condition. The form of $f_{\gamma}$ for the ES BGK model can be expressed as,

$$
f_{\gamma}=a \exp \left(-\Gamma_{i j} c_{i} c_{j}+\gamma_{i} c_{i}\right)
$$

where repeated indices indicate a sum. As in the BGK form, the coefficients are derived by satisfying conservation laws for mass, momentum and stress tensor as,

$$
\begin{aligned}
& m \int f_{\gamma} d^{3} \vec{c}=\rho, \quad m \int c_{i} f_{\gamma} d^{3} \vec{c}=\rho c_{0 i}, \\
& \frac{m}{2} \int c_{i}^{\prime} c_{j}^{\prime} f d^{3} \vec{c}=\frac{1}{\operatorname{Pr}} \frac{3}{2} \rho R T \delta_{i j}+\left(1-\frac{1}{\operatorname{Pr}}\right) \frac{m}{2} \int c_{i}^{\prime} c_{j}^{\prime} f_{\gamma} d^{3} \vec{c} .
\end{aligned}
$$

\section{BOUNDARY CONDITIONS}

The boundary conditions imposed on the domain in the simulation of a single interface are shown in Fig. 1. In order to estimate the temperature jump at the interface, known temperatures are imposed on the top and bottom boundaries of the solid and gas as shown. In the simulation of a nanoporous solid film, temperature boundary conditions are imposed on top and bottom of the domain while symmetry is imposed in the transverse direction as shown in Fig. 10. The treatment of interface and the boundary conditions is described below.

\section{A. Given-temperature boundaries \\ 1. Solid phase}

For a boundary with given temperature $T=T_{b}$, the energy density of all wave vector directions entering the domain from the boundary $(\hat{\mathbf{s}} \cdot \hat{\mathbf{n}} \leq 0)$ is considered uniform ${ }^{28}$ and is given by

$$
e^{\prime \prime}=e^{0}=\frac{C_{p}}{4 \pi}\left(T_{b}-T_{\mathrm{ref}}\right) .
$$

Here, $\hat{\mathbf{n}}$ is the outward-pointing normal from the domain and $T_{\text {ref }}$ is a reference temperature which determines the energy datum. The volumetric specific heat $C_{p}$ is assumed to be constant because the temperature difference $\left(T_{1}-T_{2}\right)$ is assumed small (Fig. 1). For all directions outgoing from the domain, the following boundary condition is used (since the incoming distribution is determined by the internal domain along the ray direction).

$$
\nabla e^{\prime \prime} \cdot \hat{\boldsymbol{s}}=0 \text {. }
$$

\section{Gas phase}

For the Maxwell-diffuse model of gas-surface interaction, the distribution function of gas molecules leaving a wall with a constant temperature is a Maxwellian given as,

$$
f_{b}=n\left(2 \pi R T_{b}\right)^{-3 / 2} e^{-\left|\overrightarrow{\mathbf{c}^{\prime}}\right|^{2} / 2 R T_{b}},
$$

where $\overrightarrow{c^{\prime}}$ is the thermal velocity of the molecules, $T_{b}$ is the temperature of the boundary, $n$ is the wall number density of 
the gas molecules at the wall, and $R$ is the universal gas constant. However, finite discretization in the velocity space can lead to considerable error in the calculation of macro parameters such as local number density and fluid velocity. The boundary conditions also must therefore be discretely satisfied as (the integrals are implemented as discrete sums),

$$
\begin{aligned}
& \int_{\hat{\mathbf{s}} \cdot \hat{\mathbf{n}}>0} c(\hat{\mathbf{s}} \cdot \hat{\mathbf{n}}) f_{b} d^{3} \vec{c}+\int_{\hat{\mathbf{s}} \cdot \hat{\mathbf{n}}<0} c(\hat{\mathbf{s}} \cdot \hat{\mathbf{n}}) f_{g} d^{3} \vec{c}=0, \\
& m \int\left(c_{i}-c_{b i}\right) f_{b} d^{3} \vec{c}=0, \\
& m \int\left(\frac{c^{\prime 2}}{2} f_{b}-\frac{3}{2} R T_{b}\right) d^{3} \vec{c}=0,
\end{aligned}
$$

where $f_{b}$ is the distribution function for gas molecules outgoing from the boundary and is a perturbed Maxwellian of the form in Eq. (3), $c_{b i}$ is the $i$ th component of the solid boundary velocity, and $T_{b}$ is the boundary temperature. For gas molecules incoming to the boundary a condition similar to Eq. (8) is used.

\section{B. Symmetry boundaries}

A symmetry boundary for either phonons or gas molecules is equivalent to specular reflection at those boundaries. For phonons all directions incoming to the domain $(\hat{\mathbf{s}} \cdot \hat{\mathbf{n}} \leq 0)$,

$$
e^{\prime \prime}(\hat{\boldsymbol{s}}, \boldsymbol{r})=e^{\prime \prime}\left(\hat{\boldsymbol{s}}_{\boldsymbol{r}}, \boldsymbol{r}\right)
$$

Similarly, for molecules for directions incoming to the domain from the boundary $(\hat{\mathbf{s}} \cdot \hat{\mathbf{n}} \leq 0)$,

$$
f_{g}(\hat{\boldsymbol{s}}, \boldsymbol{r})=f_{g}\left(\hat{\boldsymbol{s}}_{\boldsymbol{r}}, \boldsymbol{r}\right)
$$

where $\hat{\boldsymbol{s}}_{\boldsymbol{r}}$ is the incoming specular direction corresponding to $\hat{\boldsymbol{s}}$

$$
\hat{\boldsymbol{s}}=\hat{\boldsymbol{s}}_{r}-\left(2 \hat{\boldsymbol{s}}_{r} \cdot \hat{\boldsymbol{n}}\right) \hat{\boldsymbol{n}}
$$

\section{Interface between solid and gas}

At an interface between gas and solid in the presence of heat exchange, the net phonon energy flux at the interface must exactly balance the net energy flux to the gas molecules at the interface. This balance is shown in the schematic in Fig. 2. The net phonon energy flux leaving the interface is the difference of the incoming phonon flux, the reflected phonon flux and the phonon emission at the interface due to the interface temperature. We assume diffuse emission of phonons and that the emissivity of phonons is equal to $\alpha_{p}$, the absorptivity of phonons at the interface. This assumption is necessary to achieve isothermal solutions under isothermal boundary conditions. Therefore, the net energy flux leaving the interface is

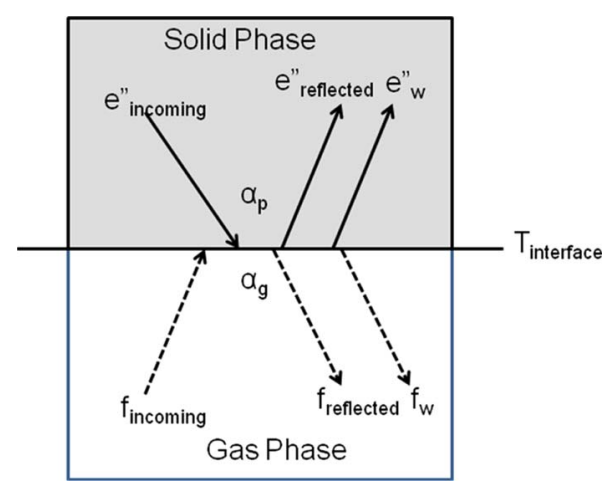

FIG. 2. (Color online) Schematic of gas-surface interaction.

$$
Q_{\mathrm{ph}}=\alpha_{p}\left(\int_{\hat{\mathbf{s}} \cdot \hat{\mathbf{n}}>\mathbf{0}} v_{g} e^{\prime \prime} \hat{\mathbf{s}} \cdot \hat{\mathbf{n}} d \Omega+\int_{\hat{\mathbf{s}} \cdot \hat{\mathbf{n}} \leq \mathbf{0}} v_{g} e_{\text {interface }}^{\prime \prime} \hat{\mathbf{s}} \cdot \hat{\mathbf{n}} d \Omega\right) .
$$

Here, $\hat{\mathbf{n}}$ is the normal vector pointing outward from the interface into the gas. Furthermore, because phonon emission from the boundary is assumed diffuse, the outgoing $(\hat{\mathbf{s}} \cdot \hat{\mathbf{n}}$ $\leq 0$ ) interface energy density for directions pointing from the interface into the solid is uniform. The energy density of phonons emitted from the interface is evaluated at $T_{\text {interface }}$, the interface temperature. For all directions incoming to the interface,

$$
\nabla e^{\prime \prime} \cdot \hat{\boldsymbol{s}}=0
$$

The net energy flux from the interface to the gas molecules as calculated on the gas side may be expressed as,

$$
\begin{aligned}
Q_{g}= & \frac{m}{2} \alpha_{g}\left[\int_{\hat{\mathbf{s}} \cdot \hat{\mathbf{n}}>\mathbf{0}} c(\hat{\mathbf{s}} \cdot \hat{\mathbf{n}}) f c^{2} d^{3} \vec{c}\right. \\
& \left.+\int_{\hat{\mathbf{s}} \cdot \hat{\mathbf{n}} \leq \mathbf{0}} c(\hat{\mathbf{s}} \cdot \hat{\mathbf{n}}) f_{\text {interface }} c^{2} d^{3} \vec{c}\right] \\
f_{\text {interface }} & =n_{\text {interface }}\left(2 \pi R T_{\text {interface }}\right)^{-3 / 2} e^{-\left|\vec{c}^{\prime}\right|^{2} / 2 R T_{\text {interface }}}
\end{aligned}
$$

Here $f$ is the incoming distribution of gas molecules, and a Maxwell model for gas-surface interaction has been used. ${ }^{50}$ According to this model, a fraction $\left(1-\alpha_{g}\right)$ of gas molecules are assumed to be specularly reflected at the interface while the remaining $\alpha_{g}$ are remitted diffusely from the interface with a Maxwellian distribution corresponding to the interface temperature $T_{\text {interface }}$ and number density $n_{\text {interface }}$ Equations (14)-(16) along with Eq. (17) to enforce mass conservation are sufficient to determine $T_{\text {interface }}$ and subsequently $f_{\text {interface }}$ and $e^{0}{ }_{\text {interface }}$ completely. Mass conservation of gas molecules at the interface may be expressed as,

$$
\begin{aligned}
& \int_{\hat{\mathbf{s}} \cdot \hat{\mathbf{n}}>\mathbf{0}} c(\hat{\mathbf{s}} \cdot \hat{\mathbf{n}}) f_{g} d^{3} \vec{c}=\alpha_{g} \int_{\hat{\mathbf{s}} \cdot \hat{\mathbf{n}} \leq \mathbf{0}} c(\hat{\mathbf{s}} \cdot \hat{\mathbf{n}}) f_{\text {interface }} d^{3} \vec{c}+(1 \\
& \left.-\alpha_{g}\right) \int_{\hat{\mathbf{s}} \cdot \hat{\mathbf{n}} \leq \mathbf{0}} c(\hat{\mathbf{s}} \cdot \hat{\mathbf{n}}) f_{\text {incoming }} d^{3} \vec{c} .
\end{aligned}
$$

To strictly satisfy conservation laws in the discretized veloc- 
ity space, the following nonlinear equation set is solved for the distribution function $f_{\text {interface }}$ of gas molecules [of the form expressed in Eq. (3)] and energy intensity $e_{\mathrm{s}}^{\prime \prime}$ of phonons leaving the interface,

$$
\begin{aligned}
& \int_{\hat{\mathbf{s}} \cdot \hat{\mathbf{n}}>\mathbf{0}} c(\hat{\mathbf{s}} \cdot \hat{\mathbf{n}}) f_{\text {interface }} d^{3} \vec{c}+\int_{\hat{\mathbf{s}} \cdot \hat{\mathbf{n}}<\mathbf{0}} c(\hat{\mathbf{s}} \cdot \hat{\mathbf{n}}) f_{g} d^{3} \vec{c}=0, \\
& \int\left(c_{i}-c_{\text {interface }}\right) f_{\text {interface }} d^{3} \vec{c}=0, \\
& m \int\left(\frac{c^{\prime 2}}{2} f_{\text {interface }}-\frac{3}{2} R T_{\text {interface }}\right) d^{3} \vec{c}=0, \\
& m \alpha_{g}\left[\int_{\hat{s} \cdot \hat{\mathbf{n}}>\mathbf{0}} c(\hat{\mathbf{s}} \cdot \hat{\mathbf{n}}) f_{\text {interface }} \frac{c^{2}}{2} d^{3} \vec{c}\right. \\
& \left.\quad+\int_{\hat{\mathbf{s}} \cdot \hat{\mathbf{n}}<\mathbf{0}} c(\hat{\mathbf{s}} \cdot \hat{\mathbf{n}}) f_{g} \frac{c^{2}}{2} d^{3} \vec{c}\right] \\
& \quad=\alpha_{p}\left(\int_{\hat{\mathbf{s}} \cdot \hat{\mathbf{n}}>\mathbf{0}} v_{g} e_{\text {interface }}^{\prime \prime} \hat{\mathbf{s}} \cdot \hat{\mathbf{n}} d \Omega+\int_{\hat{\mathbf{s}} \cdot \hat{\mathbf{n}}<\mathbf{0}} v_{g} e^{\prime \prime} \hat{\mathbf{s}} \cdot \hat{\mathbf{n}} d \Omega\right), \\
& e_{\text {interface }}^{\prime \prime}=\frac{C_{p}\left(T_{\text {interface }}-T_{\text {ref }}\right)}{4 \pi} .
\end{aligned}
$$

The balance of energy flux expressed by the equations above is used to determine the unknown $T_{\text {interface }}$ and the coefficients $\Gamma, \gamma_{x}, \gamma_{y}, \gamma_{z}\left[f_{\text {interface }}\right.$ is of the form in Eq. (3)].

\section{CALCULATION OF MACROSCOPIC PROPERTIES}

Once the values of $e^{\prime \prime}(\hat{\mathbf{s}}, \mathbf{r})$ are found, a value of solid "temperature" associated with the net phonon energy is recovered using

$$
\frac{C\left[T(\boldsymbol{r})-T_{\mathrm{ref}}\right]}{4 \pi}=e^{0}(\boldsymbol{r})=\frac{1}{4 \pi} \int_{4 \pi} e^{\prime \prime}(\hat{\mathbf{s}}, \boldsymbol{r}) d \Omega .
$$

Though the temperature $T(\mathbf{r})$ should not be interpreted as the thermodynamic temperature except under thermodynamic equilibrium, it serves as a measure of phonon energy and is useful in interpreting results.

Local fluid properties such as number density, momentum and temperature may also be computed once the field $f_{g}(c, s, r)$ is computed as,

Gas Density: $\rho=m \int f_{g} d^{3} \vec{c}$,

Bulk velocity: $c_{0 i}=\int c_{i} f_{g} d^{3} \vec{c}$,

Pressure Tensor: $P_{i j}=\int\left(c_{i}-c_{0 i}\right)\left(c_{j}-c_{0 j}\right) f_{g} d^{3} \vec{c}$,

Temperature: $T=\frac{1}{3 R_{g}} \int_{0}^{\infty} \int_{0}^{2 \pi}\left(c_{i}-c_{0 i}\right)\left(c_{i}-c_{0 i}\right) f_{g} d^{3} \vec{c}$,
Scalar Pressure: $P=\rho R_{g} T$.

\section{NUMERICAL METHOD}

The Boltzmann equations for both the gas phase distribution function and the energy density of phonons are solved using the finite volume method described in Refs. 28, 43, and 48. In addition, for the simulation of gas-phase Boltzmann equation, the velocity magnitude space is discretized using the quadrature specified in Ref. 51 with 16 abscissae.

\section{A. Calculation of the anisotropic Gaussian $\boldsymbol{f}_{\gamma}$}

After every sequential iteration over the velocity, angular, and physical spaces, the nonlinear equation set specified by Eqs. (4), (6), (10), and (18) must be solved to calculate the equilibrium values of the distribution functions $f_{g}$. The discrete versions of these equations are solved using a Newton algorithm, similar to Ref. 43. Computational effort may be significantly reduced by using reduced distribution functions when dealing with physical problems that are spatially 1D or 2D. Appendix shows the implementation for 2D problems when the ordinate associated with the polar angle may be removed altogether using reduced distribution functions $f_{1}$ and $f_{2}$.

\section{THERMAL TRANSPORT ACROSS A SINGLE INTERFACE}

We first present simulations of heat conduction in the domain shown in Fig. 1. Nondimensionalizing the governing equations and boundary/interface conditions yields the following dimensionless parameters:

$$
\mathrm{Kn}_{p}=\frac{v_{g} \tau}{L_{\mathrm{ref}}} ; \quad \mathrm{Kn}_{g}=\frac{\sqrt{2 R_{g} T_{\mathrm{ref}}}}{\nu_{\mathrm{ref}} L_{\mathrm{ref}}} ; \alpha_{p} ; \alpha_{g} ; k^{*}=\frac{k_{\mathrm{gas}}}{k_{\text {solid }}} .
$$

Here $\mathrm{Kn}_{p}$ is the Knudsen number for phonons, defined as the ratio mean free path of phonons to the reference length scale. $\mathrm{Kn}_{g}$ is the mean Knudsen number for gas molecules. These Knudsen numbers represent the degree of rarefaction in the solid and gas, respectively. The terms $\alpha_{p}$ and $\alpha_{g}$ are the interface accommodation coefficients of phonons and gas molecules, respectively, and represent the influence of detailed molecular interactions at the interface on thermal transport. The term $k^{*}$ is the ratio of bulk thermal conductivities of the gas and solid phases which, for the ES-BGK model, are related to the collision frequency of gas molecules and the mean free path of phonons as, ${ }^{49}$

$$
k_{\mathrm{gas}}=\frac{1}{\operatorname{Pr}} \frac{5}{2} R \frac{n k T}{\nu}=\frac{1}{\operatorname{Pr}} \frac{5}{2} R \mu ; \quad k_{\text {solid }}=\frac{C_{p} v_{g}^{2} \tau}{3} .
$$

It is customary to calculate the local Knudsen number for the gas phase based on the local fluid viscosity $\mu$, temperature, and pressure, and this definition for the will be employed henceforth, 


$$
\mathrm{Kn}_{g}=\frac{\mu}{p L_{\mathrm{ref}}} \sqrt{\frac{\pi}{2} R T} .
$$

We sequentially investigate the effects of the governing nondimensional parameters on heat transfer between the solidgas boundary and on the interfacial thermal resistance. Most of the simulations presented here assume perfect accommodation of gas molecules with the interface and a phonon absorptivity of unity, i.e., $\alpha_{p}=\alpha_{g}=1.0$ although the variation with these parameters is also investigated at the end of Sec. VI. A uniform structured mesh is used for all the simulations. Mesh independence tests were conducted for the case of $\mathrm{Kn}_{g}=1, \mathrm{Kn}_{p}=1$, and $k^{*}=0.001$ using meshes of 20, 50, and 100 cells. It was found that the computed thermal resistance did not change by more than $0.1 \%$ between the 50 cell and 100 cell meshes. Most simulations presented in this paper are therefore obtained using a 50 cell mesh, though finer meshes (up to 200 cells for $\mathrm{Kn}<0.1$ ) are used at lower values of $\mathrm{Kn}_{g}$ and $\mathrm{Kn}_{p}$. A uniform angular discretization of $4 \times 4$ control angles in the octant is used.

\section{A. Temperature profiles}

For verification purposes, we first compute thermal transport in domains containing only one phase. Figure 3 plots the dimensionless temperature $\theta=\left(T-T_{2}\right) /\left(T_{1}-T_{2}\right)$ with $y^{*}=y / L_{\text {ref. }}$. Figure $3($ a) shows the temperature profiles for phonon transport in a slab subject to a thermal gradient. Figure 3(b) shows heat transfer in a gas confined between two parallel plates with fixed temperature boundaries. The temperature profiles in these two cases are governed only by the corresponding Knudsen numbers. The heat transfer regimes range from the ballistic phonon transport for heat conduction and free molecular heat transfer in gases, to Fourier heat conduction in both, as can be seen from the temperature profiles in Fig. 3. As expected, in the free molecular limit $\left(\mathrm{Kn}_{g}=100.0\right)$, the temperature remains almost constant in the domain (at a value of $\sqrt{T_{1} T_{2}}$ ) and there is a sharp jump in the temperature at the two boundaries. ${ }^{42}$ The simulations were carried out with $T_{1}=301 \mathrm{~K}$ and $T_{2}=300 \mathrm{~K}$ which corresponds to $\theta=0.5$. For extremely low values of $\mathrm{Kn}_{g}$ we expect to recover Fourier conduction, including a linear temperature gradient, negligible temperature slip at the boundaries, and a heat flux corresponding to a bulk thermal conductivity of $k_{g}$. At $\mathrm{Kn}_{g}=0.01$, we do indeed recover a heat flux corresponding to the chosen value of collision frequency, $\nu$. Analogously, the phonon heat conduction problem is found to recover a constant value of temperature, $\theta=0.5$, in the domain in the ballistic limit $\left(\mathrm{Kn}_{p}=100\right)$. This value is obtained regardless of the absolute value of the temperature of the boundaries, since a linear model for phonon heat conduction has been assumed. Furthermore, at $\mathrm{Kn}_{p}=0.01$, a linear temperature gradient is observed, in keeping with the recovery of Fourier's law under bulk-scattering dominated transport. The heat flux in this limit is found to correspond to a thermal conductivity of $k_{s}=C_{p} v_{g}^{2} \tau / 3$. The analytical solution ${ }^{52}$ for the mathematically analogous problem of radiative transfer in a gray medium between parallel plates is plotted as circles in Fig. 3 along with the numerically calculated temperature pro-
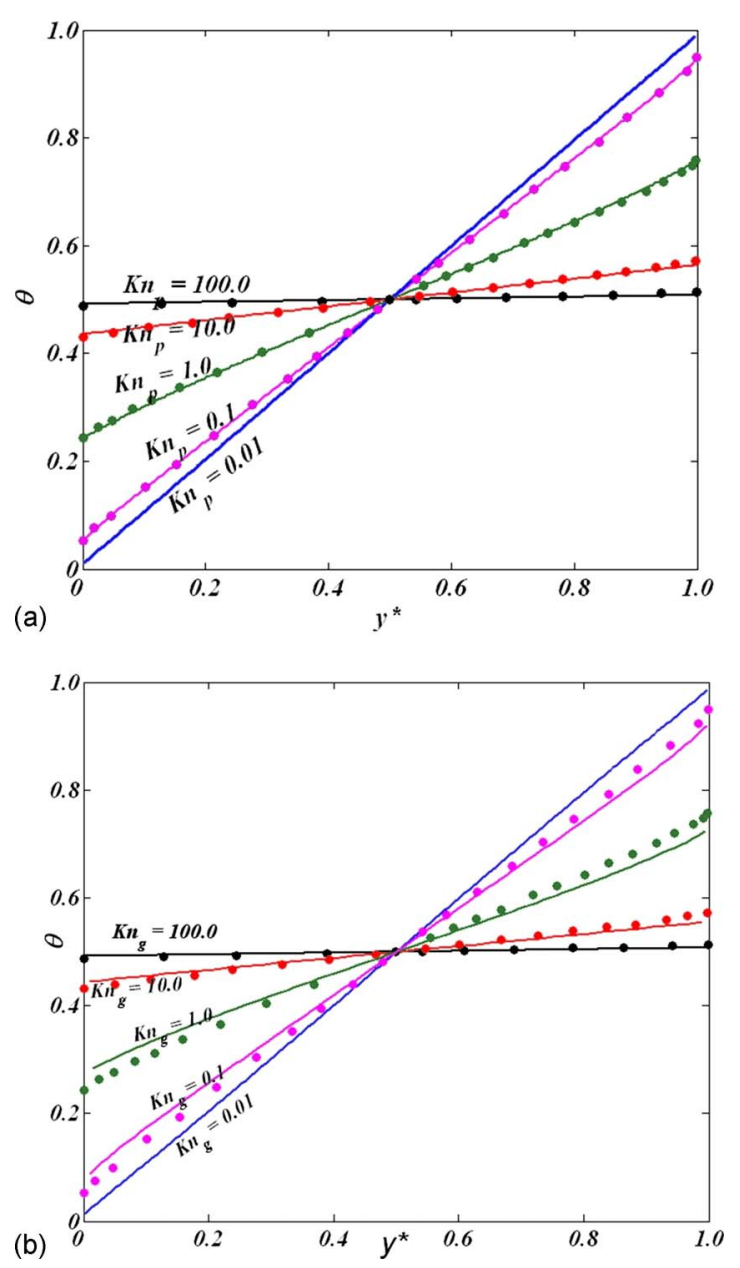

FIG. 3. (Color online) Contours of dimensionless temperature for (a) phonon heat conduction in a slab and (b) gaseous heat transfer between two parallel plates. Solid lines-numerical solution; dots-exact solution for gray BTE (Ref. 52).

files. The match with Ref. 52 is good for phonon transport, but less so for gas phase transport. This is because the solution in Ref. 52 is not analogous to the gas phase result since a single velocity assumption for molecular velocity has not been made in the latter.

Figure 4(a) shows the dimensionless temperature profiles for heat transfer between two parallel plates for various values of $\Delta T$ with $T_{1}=300 \mathrm{~K}$ and $\mathrm{Kn}_{g}=1.0$ (based on $T_{1}$ $=300 \mathrm{~K})$. The gas is assumed to be helium, with a viscositytemperature relation given by $\mu=\mu_{\text {ref }}\left(T / T_{\text {ref }}\right)^{\beta}$ with $\beta=0.66$ and $\operatorname{Pr}=2 / 3 .^{42}$ This induces a temperature-dependent local collision frequency thereby introducing nonlinear effects. This effect can be seen on temperature profiles and appears to be pronounced only for temperature differences greater than $100 \mathrm{~K}(\Delta T / T>0.3)$ or so. Figure $4(\mathrm{~b})$ shows the effect of temperature difference on the nondimensional heat flux $Q^{*}=Q /\left(\Delta T^{*} Q_{\Delta T=1}\right)$ where the denominator is the heat flux obtained with a temperature difference of $1 \mathrm{~K}$, and may be considered to be in the linear regime. While a significant difference is seen in the free molecular case, a difference of only $3 \%$ is observed at $\mathrm{Kn}_{g}=1.0$.

Figure 5 shows the dimensionless temperature profiles for a gas-solid interface with $L_{g}=L_{\text {ref }} / 2$. The temperature profiles are shown for various values of $k^{*}$ in the mesoscopic 

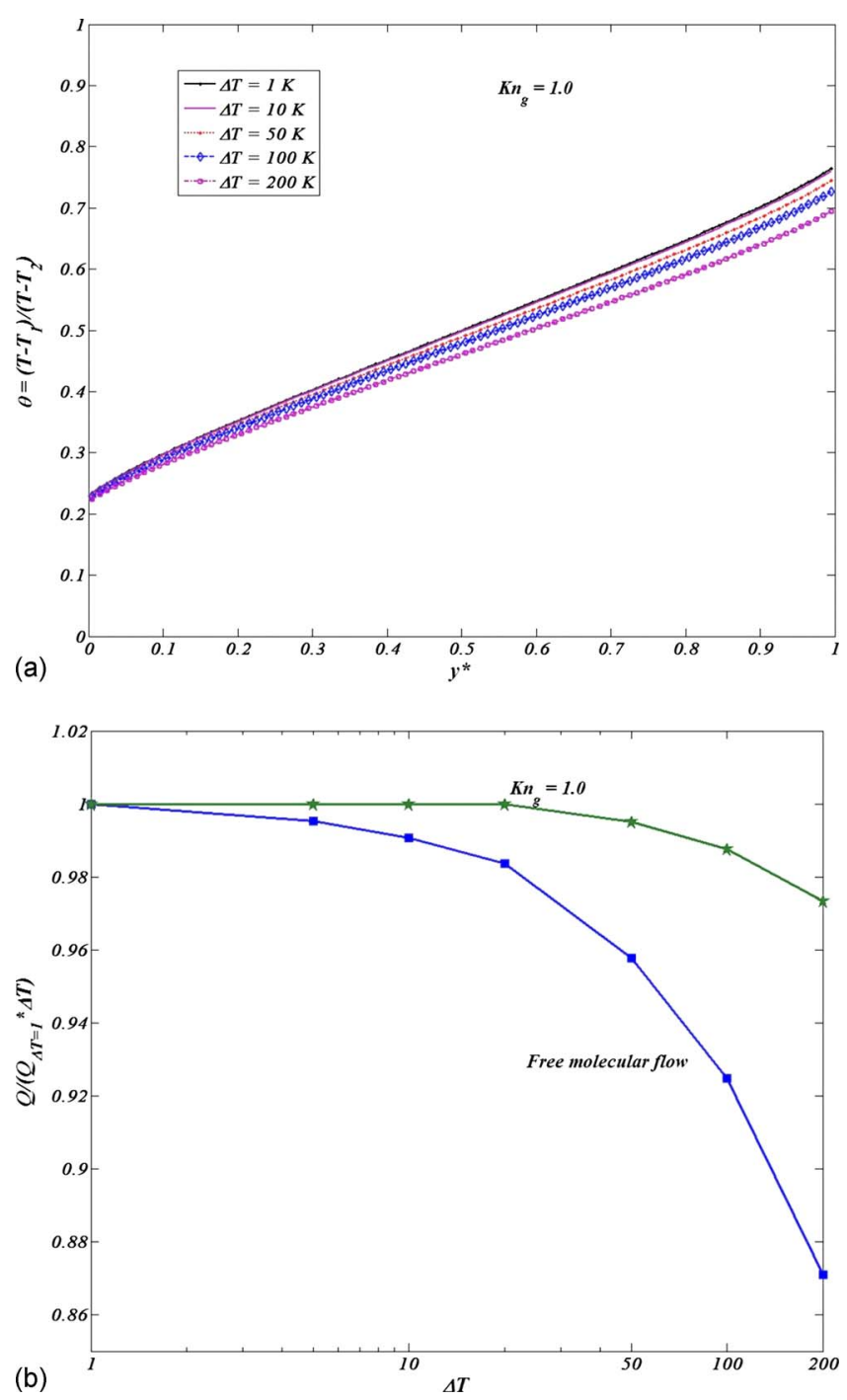

FIG. 4. (Color online) Nonlinear thermal effects in (a) dimensionless temperature profile along $y^{*}$ for $\mathrm{Kn}_{g}=1.0$ for different values of $\Delta T / T$; (b) dimensionless heat flux vs $\Delta T$.

regime $\left(\mathrm{Kn}_{g}, \mathrm{Kn}_{p}=1.0\right.$ and $\left.\mathrm{Kn}_{g}, \mathrm{Kn}_{p}=0.1\right)$ where analytical expressions are limited and an accurate solution of the Boltzmann equation is necessary. For interfaces between solid silicon and air, for example, $k^{*} \sim 0.0001$, no observable temperature drop is expected in the bulk of the solid and most of the temperature gradient occurs on the gas side. There is however a sharp drop in the temperature at the gas-solid interface. At moderate values of $\mathrm{Kn}(\sim 1)$ this temperature jump is due primarily to rarefaction effects in both phonon transport and gas phase transport. Significant temperature drops in both the solid and at the interface occur for $k^{*}$ $=0.1$ or greater. This low value of $k^{*}$ corresponds to interfaces between low conductivity solids such as oxides, polysilicon, and high conductivity gases such as $\mathrm{H}_{2}$ and $\mathrm{He}$. At $\mathrm{Kn}=0.1$, most of the temperature drop occurs in the bulk of the gas and solid, with a small temperature jump at the boundaries.

\section{B. Interfacial thermal resistance}

Once the temperature field is computed, the temperature drop across the gas-solid interface is determined from,
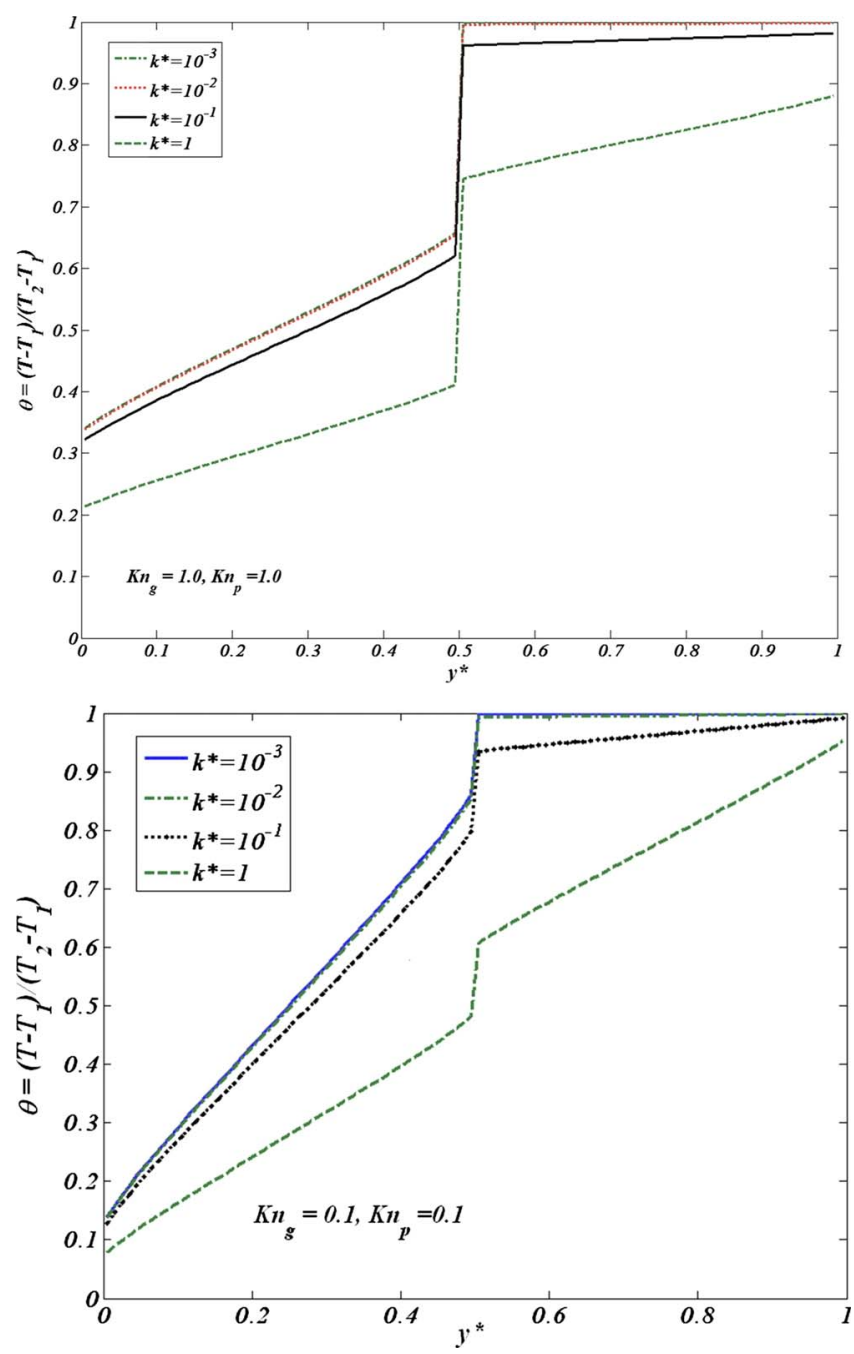

FIG. 5. (Color online) Dimensionless temperature profile along $y^{*}$ with a gas-solid interface for $\mathrm{Kn}_{g}=\mathrm{Kn}_{p}=1.0$ (top) and $\mathrm{Kn}_{g}=\mathrm{Kn}_{p}=0.1$ (bottom).

$$
\Delta T_{\text {jump }}=T\left(y_{\text {phonons }}^{+}\right)-T\left(y_{\text {gas }}^{-}\right) .
$$

Here, $T\left(y^{+}\right.$phonons $)$corresponds to the temperature extrapolated from the bulk solid to the interface, while $T\left(y_{\text {gas }}^{-}\right)$represents the interface temperature extrapolated from the bulk gas. The interface resistance is calculated as,

$$
R_{\text {jump }}=\Delta T_{\text {jump }} / Q \text {. }
$$

The results presented here are normalized values of interfacial resistance, defined as

$$
\begin{aligned}
R^{*}= & \frac{R_{\text {jump }}}{\left(T_{1}-\sqrt{T_{1} T_{2}}\right) / Q_{\mathrm{FM}}}, \quad Q_{\mathrm{FM}}=\frac{2^{3 / 2}}{\sqrt{\pi}}\left(p_{1} \sqrt{R T_{1}}\right. \\
& \left.-p_{2} \sqrt{R T_{2}}\right)
\end{aligned}
$$

where $p_{1}, p_{2}$ correspond to gas pressures at plate 1 and plate 2 , respectively, $Q_{\mathrm{FM}}$ represents the heat flux for free molecular flow between the plates, and the reference temperature jump is given by $\left(T_{1}-\sqrt{T_{1} T_{2}}\right)$. For an internally confined gas, fixing $T_{1}$ and $T_{2}$, and pinning the initial mass density of the gas based on a pressure of $1 \mathrm{~atm}$ self consistently determines $p_{1}$ and $p_{2}$ through the ideal gas law and conservation of mass (which can be analytically calculated for free molecular 


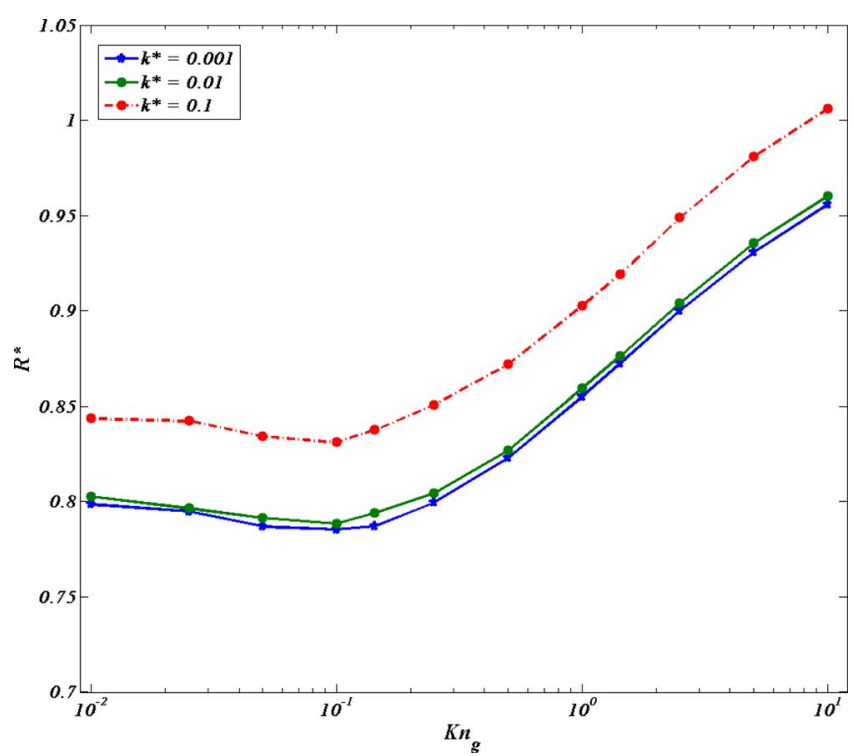

FIG. 6. (Color online) Resistance as a function of $\mathrm{Kn}_{g}$ and $\Delta T / T$ $=0.003 \mathrm{~K}$.

cases ${ }^{42}$ ). All reported simulations in this paper assume a nominal gas pressure of 1 atm.

We investigate the values of interfacial thermal resistance as a function of the Knudsen number for gas molecules. For convenience, constant ratio of $\mathrm{Kn}_{g} / \mathrm{Kn}_{p}=1.0$ is used in all simulations in keeping with the fact that average mean free paths for phonons in the solid are comparable to mean free path of gas molecules at atmospheric pressure. A monatomic gas (with no internal energy) with Prandtl number of one is assumed for all the calculations presented in the following sections.

\section{Effect of gas phase Knudsen number $\mathrm{Kn}_{\mathrm{g}}$}

Figure 6 illustrates the dependence of the interfacial resistance on the gas phase Knudsen number at $\mathrm{Kn}_{g} / \mathrm{Kn}_{p}$ $=1.0$ for various values of $k^{*}$. There is a mild dependence of the interfacial thermal resistance on $\mathrm{Kn}_{g}$. For low values of $k^{*}$, as $\mathrm{Kn}_{g} \rightarrow \infty$, the resistance approaches that for free molecular flow. However it approaches a constant value $\sim 0.8$ as $\mathrm{Kn}_{g} \rightarrow 0$. This may be understood in terms of Eq. (26), which predicts the temperature jump in the gas phase in the slip regime $\left(\mathrm{Kn}_{g}<0.1\right)$. For $\mathrm{Kn}<0.1$, the problem may be treated under the framework of the classical diffusive heat conduction equation with a bulk thermal conductivity and using the temperature slip boundary condition (see, for example, Refs. 53 and 54),

$$
T_{\text {slip }}=T_{\text {wall }}+\frac{2-\alpha_{g}}{\alpha_{g}} \frac{2 \gamma}{\gamma+1} \frac{2 \mu}{\rho} \sqrt{\frac{\pi}{8 R T}} \frac{1}{\operatorname{Pr}}\left(\frac{\partial T}{\partial y}\right)_{\text {slip }} .
$$

For an ideal monatomic gas with perfect accommodation at a solid surface this reduces to

$$
T_{\text {slip }}=T_{\text {wall }}+\frac{1.25 \lambda_{g}}{\operatorname{Pr}}\left(\frac{\partial T}{\partial y}\right)_{\text {slip }},
$$

where, $\lambda_{g}$ is the mean free path of the gas the corresponding $\mathrm{Kn}$ for which is calculated as pointed in Eq. (23). The tem- perature slip given by Eq. (26) is derived under the assumption that the distribution function of molecules is constant within the Knudsen layer adjacent to solid walls. However, when the value of temperature slip is used as the boundary condition for classical diffusive heat conduction in the gas phase, it reproduces the correct temperature distribution outside the Knudsen layer at small Knudsen numbers, typically $\mathrm{Kn}<0.05$. The resistance in this limit approaches a constant value of $1.25 \lambda_{g} /\left(\operatorname{Pr} k_{g}\right)$ for a monatomic gas. For the cases considered here (monoatomic gas in the linear regime $\left(T_{2}\right.$ $\left.-T_{1}\right) \rightarrow 0$ ), the normalized value of this resistance is 1 . However, our calculations reveal a lower limit near 0.8 for $k^{*}$ $\rightarrow 0$. This difference may be attributed to our use of the temperature value extrapolated to the wall, whereas under a slip approximation this value corresponds to that at the edge of the Knudsen layer, about one mean free path away from the wall. ${ }^{66}$ As $k^{*}$ increases, the interfacial resistance exhibits a dependence on $k^{*}$. The resistance exhibits values greater than 1.0 as $\mathrm{Kn}_{g} \rightarrow \infty$ since the solid phase now offers a significant resistance to heat flow. An asymptote of about $R^{*}$ $=0.85$ is achieved for $k^{*}=0.1$ for $\mathrm{Kn}_{g} \rightarrow 0$; this higher value is also attributed to non-negligible thermal resistance being offered by the solid phase.

Notably, the value of interfacial thermal resistance does not change significantly with changes in Knudsen number. However it becomes a significant fraction of the overall thermal resistance as Knudsen number increases. In the limit of free molecular flow/ballistic phonon transport, the temperature drop across the bulk of the solid and gas is negligible since molecules and phonons exhibit a free flight between boundaries; the main temperature drop is across the interface. In this limit, the interfacial thermal resistance completely controls the temperature drop across the domain. This limit is also has significant impact for applications such as nanopowders and cantilever tips where characteristic lengths are smaller than phonon and gas mean free paths.

The results of the Sec. VI B bring us to the important question of finding the value of $k^{*}$ beyond which phonon transport in the solid determines the temperature jump at the interface and therefore, the interfacial thermal resistance. For most commonly encountered material combinations, $k^{*}$ is low, and the interface resistance is governed by the gas side, as expected, leading to a constant resistance value dependent only on gas properties and $\mathrm{Kn}_{g}$. However, beyond $k^{*}=0.01$, typical of the interfaces of polycrystalline silicon or glass with high conductivity gases such as $\mathrm{He}$ and $\mathrm{H}_{2}$, the value of interface resistance increases significantly with $k^{*}$ in the mesoscopic regime. Near the diffuse limit, however, $\left(\mathrm{Kn}_{g}\right.$ $\sim \mathrm{Kn}_{p} \sim 0.1$ ) values of interfacial thermal resistance are found to be almost independent of the thermal conductivity ratio.

\section{Effect of $\alpha_{p}$ and $\alpha_{g}$ : Phonon absorptivity and accommodation coefficients}

All the results presented in the previous sections assume perfect accommodation of gas molecules to a solid surface and a phonon absorptivity of one. This condition represents a theoretical maximum for the values of interfacial heat flux 
and also leads to the lowest temperature slip at the boundary. This is evident from Eq. (26), which shows that temperature slip scales inversely as $\alpha_{g}$. Equations (14)-(17) demonstrate that heat flux directly scales as the phonon absorptivity and gas surface accommodation coefficients. These parameters are therefore expected to be the primary determinants of interfacial thermal resistance at a gas-solid boundary. There has been significant effort dedicated to quantifying the accommodation of gas molecules on a solid surface both experimentally $^{11,55,67}$ and from MD simulations. ${ }^{56,57}$ While each transport property has its own accommodation to a surface, the Maxwell model for gas-surface interaction has been widely used to describe transport phenomena that do not include high speed nonisothermal conditions. In the latter case, the Cercignani-Lampis scattering kernel $^{58,59}$ finds a better match against experiments. For the particular problem of heat transfer between parallel plates, Maxwell's gas surface interaction law with a thermal accommodation coefficient $\alpha_{g}$ has been shown to adequately represent most experimental observations. ${ }^{54,55}$ In the present study, we use this particular scattering kernel to represent gas-surface interaction. The value of $\alpha_{g}$ for most engineering surfaces with air and other diatomic gases such as $\mathrm{N}_{2}$ has been reported to be close to 0.8-0.9. However, for extremely light gases such as $\mathrm{He}$ on atomically clean surfaces, values as low as 0.02 have been experimentally observed. ${ }^{55}$

Phonon transmission and scattering on rough interfaces has been a topic of ongoing research. While traditional theoretical estimates of phonon transmission in solids are often based on simple analytical acoustic mismatch or the diffuse mismatch models, ${ }^{60}$ these have failed to explain several experimental observations made recently. ${ }^{1}$ More recently, the AGF (Ref. 34) and the MD wave packet method ${ }^{61,62}$ have been used to determine phonon transmission values across the frequency spectrum. However, no estimates of this value exist for gas-solid boundaries. Because the gas is a highly rarefied medium in comparison to the solid, these values are expected to be much lower than 1.0. However the existence of certain surface phonon modes can facilitate energy transfer between the bulk phonons and gas molecules. In the present study we parameterize $\alpha_{p}$ to elucidate its effects on the thermal resistance. Figure 7 shows the values of thermal resistance as a function of $\alpha_{p}$ for four different values of $\alpha_{g}$ in the commonly encountered gas-surface combinations. In general $\alpha_{g}$ is the main determinant of interfacial thermal resistance. A modest dependence on $\alpha_{p}$ is found at moderate $k^{*}(=0.01)$, of the order of about $10 \%$. However for low conductivity solids the resistance can increase three- and fourfold as $\alpha_{p}$ values tend to 0.1 .

These results point to the necessity of considering solidphase temperature gradients in the interpretation of the temperature slip length in gases. In the measurement of slip length for confined air between a substrate and a glass sphere attached to an AFM cantilever for example, ${ }^{63}$ neglecting the temperature drop in the glass would cause the apparent temperature drop in the gaseous phase to be overestimated significantly. This discrepancy is, however, negligible when the thermal conductivity of the solid is much higher that of the gas. Similarly, in the modeling of thermal conductivity of

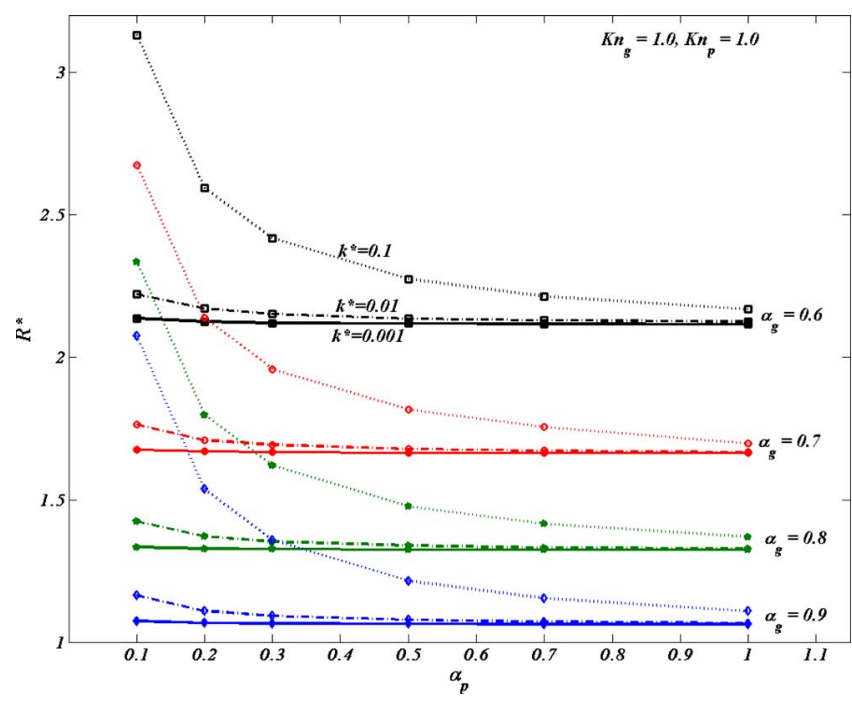

FIG. 7. (Color online) Resistance as a function of phonon absorptivity $\alpha_{p}$ and gas accommodation coefficient $\alpha_{g}$. Dashed lines show resistance for $k^{*}=0.1$, dashed-dot curves represent $k^{*}=0.01$, and solid lines represent $k^{*}$ $=0.001$.

particle beds, the thermal resistance at the interface has accounted for the slip length in the gas phase while neglecting effects of phonon transmission, ${ }^{64}$ possibly leading to erroneous results for the case when the solid phase has low thermal conductivity.

\section{E. Reduced order modeling}

The results developed in the preceding sections may be used under the framework of a 1D thermal resistance model $^{65}$ based on Fourier conduction. Consider again the physical configuration represented in Fig. 1. The usual Fourier representation of heat transfer in this domain includes temperature drop only in the bulk of the solid $\left(L_{s}\right)$ and the gas $\left(L_{g}\right)$ to give a resistance,

$$
R=\frac{L_{g}}{k_{g}}+\frac{L_{s}}{k_{s}} .
$$

As the length $L$ decreases, this predicts $R \rightarrow 0$. However, physically, the heat transfer rate must still be finite due to the finite velocity of heat carriers. These physics may be captured by including interface and boundary resistances into the Fourier representation.

The temperature drop across a constant-temperature gas boundary may be calculated as in Eq. (26). As pointed out earlier this is equivalent to a linearized locally free molecular assumption and therefore leads to a nondimensional resistance value of $R^{*}=1.0$. Similarly, the temperature jump at a given temperature boundary for phonons under the gray assumption may be calculated based on the basis of ballistic phonon transport to be $R=2 / C v_{g}$ which is nondimensionalized based on Eq. (25). These thermal boundary resistance (TBR) values and the interfacial thermal resistance at the gas-solid interface may now be included in the resistance model to represent finite resistance even as $L \rightarrow 0$. This is illustrated schematically in Fig. 8.

Figure 9 shows the value of total thermal resistance between the two boundaries, defined as $\left(T_{1}-T_{2}\right) / Q$, where $Q$ is 


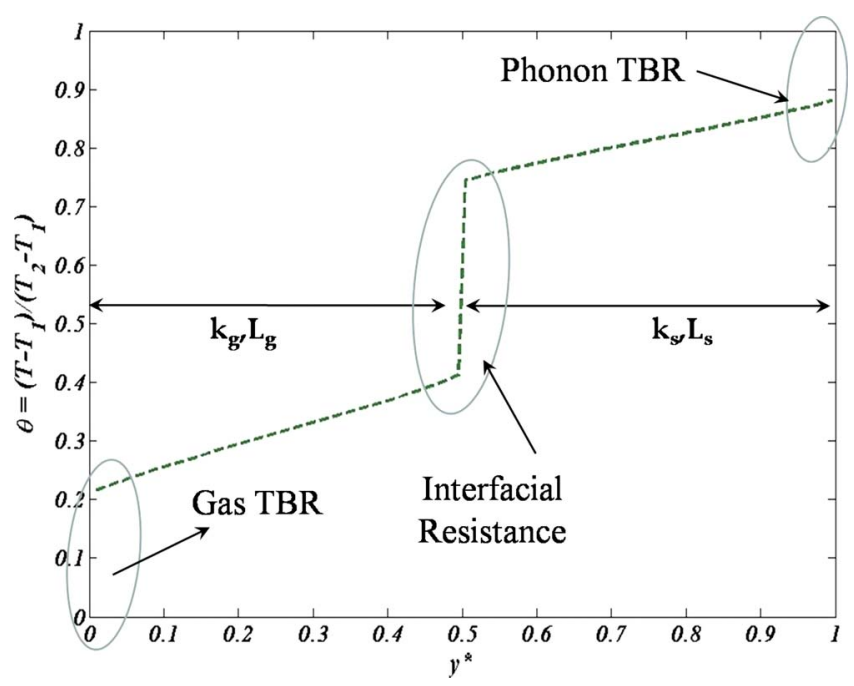

FIG. 8. (Color online) Schematic of the modified Fourier model including TBR and interfacial thermal resistance.

the net heat flux rate. It differs from the resistance shown in Fig. 6 in that the total resistance accounts for temperature jump due to boundary scattering as well as the temperature drop through the bulk of the gas and the solid itself. The interfacial thermal resistance at the gas-solid boundary, on the other hand, only quantifies the temperature jump that occurs across this interface. $\boldsymbol{R}_{\mathrm{tot}}^{*}$ is calculated in three different ways in Fig. 9. The first uses a solution of the phonon and gas phase BTEs and is labeled "BTE" in Fig. 9; this is the most rigorous way of computing thermal transport across such a structure. The second uses a Fourier model, but includes the TBR and interfacial thermal resistance in addition to the bulk fluid and solid thermal resistances; this is labeled Fourier+TBR in Fig. 9. The last uses a Fourier model incorporating only bulk gas and solid thermal resistance; this is an approximate model that should give results similar to the BTE for $\mathrm{Kn}<0.01$ ), and is labeled "Fourier" in Fig. 9, These are plotted for two different values of $k^{*}$. The normalized total resistance tends to an asymptote of two as $\mathrm{Kn} \rightarrow \infty$ for

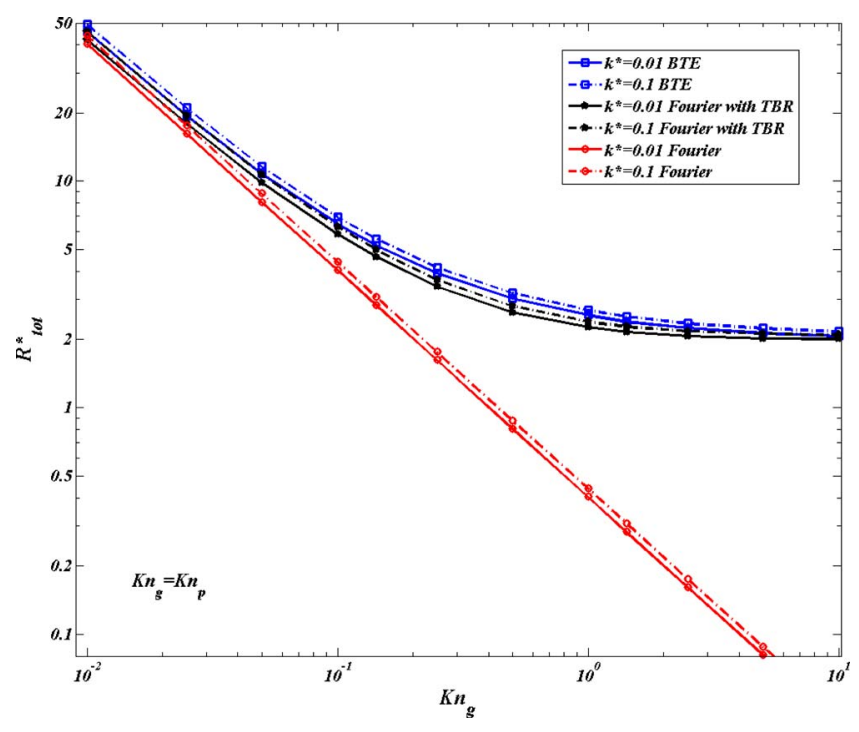

FIG. 9. (Color online) Total thermal resistance as a function of $\mathrm{Kn}_{g}=\mathrm{Kn}_{p}$.

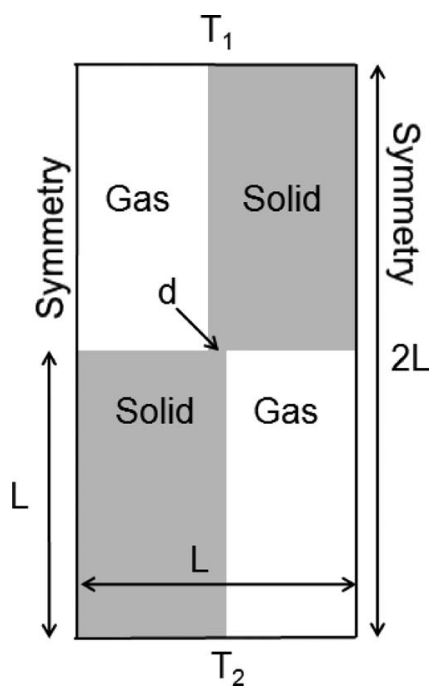

FIG. 10. Schematic of a model nanoporous film with a constricted solid contact.

the BTE and Fourier+BTE models This asymptotic value is obtained because the temperature drop is limited to only the gas-solid interface and the gas temperature boundary at high $\mathrm{Kn}$ and low $k^{*}$; the solid is nearly isothermal. Thus the interface contributes a value of $R^{*}=1.0$, and the gas-phase boundary an additional value of $R^{*}=1.0$ (The two values are equal since the solid represents an isothermal boundary condition under this limit of $k^{*}$; therefore the thermal transport properties of the solid do not impact the interfacial thermal resistance). However, the value of $R_{\text {tot }}^{*}$ predicted using the Fourier model tends to zero in the high Kn regime but asymptotically tends to the exact solution of the BTE for $\mathrm{Kn}<0.1$. In this range of $\mathrm{Kn}$, bulk scattering of gas molecules in the gas phase and bulk phonon scattering in the solid overwhelm any effects of boundary scattering and computed solutions approach those estimated by traditional diffusion theory, which predicts linear scaling of thermal resistance with the sample dimension. At $\mathrm{Kn}=0.01 R_{\mathrm{tot}}^{*}$ is as high as 50 which means that scattering of heat carriers among themselves becomes much more important than the interfacial resistance. However the Fourier model predicts the $R_{\text {tot }}^{*}$ value tends to zero for $\mathrm{Kn}>10$. It cannot describe the ballistic transport regime because it does not account for the finite thermal resistance due to boundary scattering. The inclusion of TBR, however, remedies the problem; we see that the Fourier+TBR curves fall close to the BTE curves even at high $\mathrm{Kn}$.

\section{2D HEAT CONDUCTION IN A NANOPOROUS BED}

The case of 1D heat conduction in the solid-gas geometry shown in Fig. 1 illustrates the basic mechanisms governing thermal transport in structures containing such interfaces. However heat conduction is often multidimensional in structures of practical interest. Here we illustrate the applicability of the model to heat transfer in an idealized 2D nanoporous film geometry, as shown in Fig. 10. Two solid particles of length scale $L$ make contact through a constriction of size $d$. We consider thermal transport through the particles, across the constriction and through the surrounding gas phase, which offers a parallel pathway for heat transfer. 
The solution of the Boltzmann equation for heat carriers in both the solid and gas is necessary to accurately capture size effects related to thermal transport through the constriction and quasiballistic heat transfer through the gas phase. The governing equations and boundary/interface conditions in this case yield the following dimensionless parameter set:

$$
\mathrm{Kn}_{p}=\frac{v_{g} \tau}{L} ; \mathrm{Kn}_{g}=\frac{\mu}{p L} \sqrt{\frac{\pi}{2} R T} ; \alpha_{p} ; \alpha_{g} ; k^{*}=\frac{k_{\mathrm{gas}}}{k_{\text {solid }}} ; d^{*}=\frac{d}{L} .
$$

While the basic nondimensional parameters that appear in thermal transport across a single interface $\left(\mathrm{Kn}_{p}, \mathrm{Kn}_{g}, \alpha_{p}, \alpha_{g}, k^{*}\right)$ appear here as well (with $\left.L_{\mathrm{ref}}=L\right)$, the presence of a constricted solid interface gives rise to a new length scale $d^{*}$. In addition to the physical properties of the gas and solid phase, $d^{*}$ governs the relative importance of the gas and solid pathways in determining the cross-plane thermal resistance of the structure. The Knudsen number $\mathrm{Kn}_{p}$ is calculated based on $L$ with $\mathrm{Kn}_{p}=\mathrm{Kn}_{g}$. For all simulations, a value $d^{*}=0.02$ is used, and corresponds to a $2 \%$ contact area between the two solids. The Knudsen number based on the contact width $d$ is thus 50 times that based on the particle width $L$, which implies that thermal transport through the constriction is always near ballistic for the range of $\mathrm{Kn}_{p}$ explored here (0.1-10). It should be noted here that in 3D contacts the dimensionless contact area scales as $(d / L)^{2}$ thereby magnifying the importance of gas gap conduction at 3D contacts. The simulations presented in this section assume perfect accommodation of gas molecules with the interface and a phonon absorptivity of unity, i.e., $\alpha_{p}=\alpha_{g}=1.0$. Lower values of accommodation coefficient and phonon absorptivity will lead to a higher interfacial resistance, making the gas pathway more resistive. A uniform structured mesh of 200 $\times 200$ cells is used for these simulations. Meshindependence tests were conducted for the case of $\mathrm{Kn}_{g}=0.1$, $\mathrm{Kn}_{p}=0.1, k^{*}=0.0001$, and $d^{*}=0.02$ using meshes of 100 $\times 100,200 \times 200$, and $300 \times 300$ cells, and a $200 \times 200$ cell was found yield overall heat transfer rates accurate to under $1 \%$. A uniform angular discretization of $8 \times 8$ control angles in the octant is used.

Figure 11 shows the nondimensional temperature contours overlaid with the local heat flux vectors at $\mathrm{Kn}=10$ for two different gas-solid thermal conductivity ratios, $k^{*}=0.1$ and 0.0001. Because the phonon mean free path is much greater than the solid dimension, the transport is near ballistic and dominated by boundary scattering in both the solid and at the contact. The majority of the temperature drop in the solid therefore is across the constriction, and temperature remains relatively uniform throughout the rest of the domain. At both values of $k^{*}$ there is a moderate temperature drop in the gas phase. The heat flux vectors clearly indicate that the heat conduction path is completely dominated by the solid at $k^{*}=0.0001$. On the other hand, the constriction is relatively more resistive at $k^{*}=0.1$, and significant energy exchange to the gas takes place along the interface.

The results for the net thermal resistance, $R=\left(T_{1}\right.$ $\left.-T_{2}\right) / Q$ between the top and bottom boundary as a function of gas-solid thermal conductivity ratio $k^{*}$ at $\mathrm{Kn}=0.1,1$ and
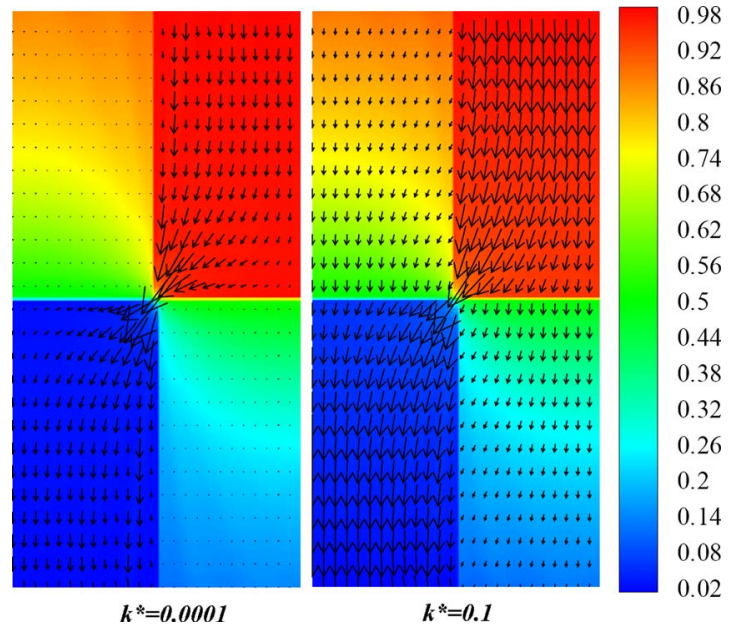

FIG. 11. (Color online) Contours of nondimensional temperature and heat flux vectors through the domain at $\mathrm{Kn}=10$.

10 (with $\mathrm{Kn}_{p}=\mathrm{Kn}_{g}$ ) are shown in Fig. 12. The plotted thermal resistance is normalized by the classical ballistic constriction resistance for phonons, so that $R^{*}=R\left(C v_{g} d / 4\right)$. In the limit of $k^{*} \rightarrow 0$ at $\mathrm{Kn}=10, R^{*}$ approaches a value slightly higher than unity. The extra resistance originates from diffuse scattering of phonons along the gas-solid interface near the constriction (with specular phonon reflection at the interface, we exactly recover the ballistic constriction resistance). However, as $k^{*}$ increases, the resistance quickly drops, and significant heat conduction occurs through the gas because of the high constriction resistance of the solid-solid contact. The resistance is significantly higher at $\mathrm{Kn}=0.1$ because of strong phonon scattering in the bulk of the solid. Although the trends in the dependence of $R$ on $k^{*}$ is similar at various $\mathrm{Kn}$, quantitative values show that the relative drop in resistance increases as $\mathrm{Kn}$ increases. The decrease in resistance between $k^{*}=0.0001$ and $k^{*}=0.1$ is almost $75 \%$ at $\mathrm{Kn}=10$ but only $45 \%$ at $\mathrm{Kn}=0.1$. This is because increases in the mean free path of the gas molecules push transport to the free

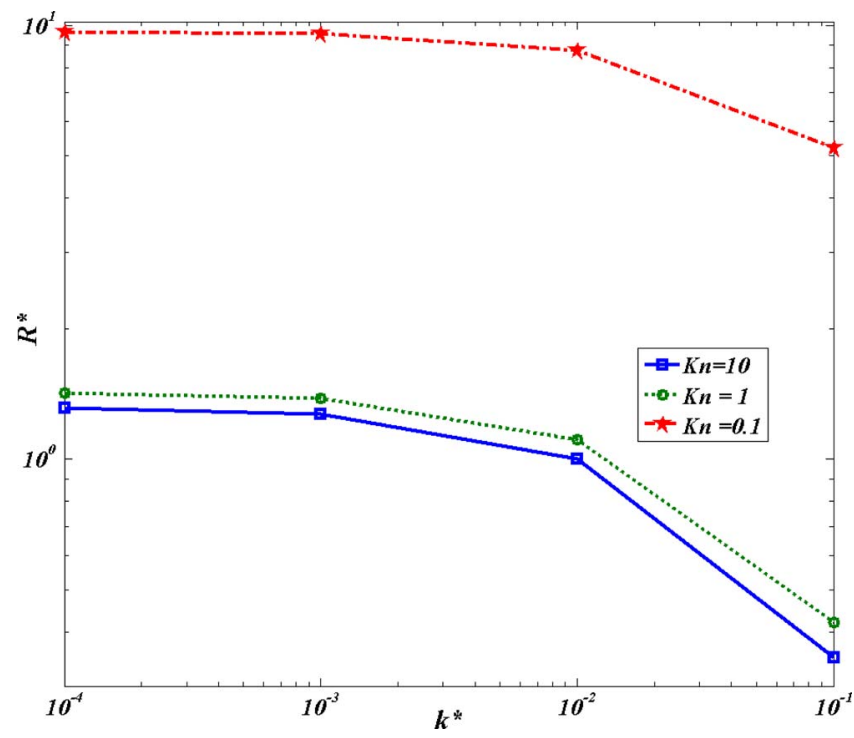

FIG. 12. (Color online) Net thermal resistance as a function of Knudsen number and gas-solid thermal conductivity ratio. 
molecular regime, decreasing the thermal resistance of the parallel gas conduction pathway. The computed thermal resistance directly relates to the effective bulk thermal conductivity of the nanoporous structure as $k_{\mathrm{eff}}=2 / R$ $=3 k_{\text {solid }} d^{*} /\left(2 \mathrm{Kn}_{p} R^{*}\right)$. It can be easily seen that for the case of $\mathrm{Kn}_{p}=1$ and $d^{*}=0.02$, the presence of a surrounding gas medium can increase the effective thermal conductivity by a factor $>3$ for $k^{*}=0.1$.

\section{CONCLUSIONS}

In this paper, a consistent simulation methodology for nanostructures involving solid-gas interfaces has been developed. The method involves direct solution of the Boltzmann equation for phonons and gas molecules using the finite volume method. The interface is assumed to attain an equilibrium temperature consistent with energy balance. The accommodation coefficient of gas molecules and the phonon transmissivity across the interface determine the degree of equilibrium established between the two carriers. The computational algorithm has been validated against the benchmark cases involving heat conduction in a slab (solid) and between two parallel plates (gas). Temperature profiles for the case of thermal transport across the interface between a semiconductor thin film and an ideal gas are computed. Values of interfacial thermal resistance are computed, and as expected, are found to be dominated by the low-conductivity gas. The crossplane thermal resistance and thermal conductivity of a 2D nanoporous solid film is calculated and found to be strongly influenced by conduction in the surrounding gas for constriction length scales of the order of the phonon mean free path. A far larger gas-phase role is expected in 3D geometries where the solid-solid constriction resistance is expected to be much larger. Though the demonstration employs idealized particles, generalization of the computational methodology to complex geometries is straightforward, and would enable simulation of heat transfer in particle beds and in other practical applications. ${ }^{68}$

The computed results depend strongly on the phonon transmission at gas/solid interfaces. Experimental determination of phonon transmissivity is a significant challenge, and MD simulations may lead to useful insights here. Transmission coefficient values are expected to be much lower than 1.0 due to a high degree of mismatch between the acoustic properties of the gas and the solid. ${ }^{1}$ The thermal resistance values reported here may be used with conventional continuum heat transfer tools to model a variety of thermal transport problems.

\section{ACKNOWLEDGMENTS}

The authors would like to acknowledge support from the Purdue Computing Research institute (CRI). D.S. and J.M. wish to acknowledge support from the DARPA-funded IMPACT Center at University of Illinois. Support from the Department of Energy (National Nuclear Security Administration) under Award No. DE-FC52-08NA28617 and Purdue Research Foundation is gratefully acknowledged.

\section{APPENDIX: REDUCED DISTRIBUTION FUNCTIONS}

For transport limited to two dimensions it is not necessary to solve for the complete distribution function. Instead we can derive reduced distribution functions that depend only on the gas velocity in the plane of transport. Assuming transport in the $x y$ plane $\left(c_{0 z}=0\right)$ we may integrate over the $z$ component of velocity $w$ to obtain,

$$
\begin{aligned}
& f_{1}=\int_{-\infty}^{\infty} f d c_{z}, \\
& f_{2}=\int_{-\infty}^{\infty} f c_{z}^{2} d c_{z} .
\end{aligned}
$$

All bulk and fluxal properties of interest may be calculated from the knowledge of these two reduced distribution functions. We shall henceforth denote the velocity magnitude in the $x y$ plane as $v$ and the associated velocity direction as $\varphi$. The latter is exactly the same as the azimuthal component of the phonon wave vector. Therefore,

$v_{x}=v \cos \varphi, \quad v_{y}=v \sin \varphi$.

This procedure reduces only one dimension in the velocity space of the Boltzmann equation. The Boltzmann equation for the reduced distribution functions may be written as,

$$
\begin{aligned}
& \nabla .\left(v \mathbf{s} f_{1}\right)=-\nu\left(f_{1}-f_{\gamma 1}\right), \\
& \nabla .\left(v \mathbf{s} f_{2}\right)=-\nu\left(f_{2}-f_{\gamma 2}\right) .
\end{aligned}
$$

For 1D transport, this procedure may be used to eliminate two components of the discrete velocity space.

The equilibrium velocity distribution function of gas molecules is a Maxwellian. Writing this in terms of reduced distribution function with $c_{0 z}=0$ we have,

$$
\begin{aligned}
& f_{1}=n\left(2 \pi R T_{b}\right)^{-1} e^{-\left[\left(v_{x}-v_{x 0}\right)^{2}+\left(v_{y}-v_{y 0}\right)^{2}\right] /\left(2 R T_{b}\right),} \\
& f_{2}=n(2 \pi)^{-1} e^{-\left[\left(v_{x}-v_{x 0}\right)^{2}+\left(v_{y}-v_{y 0}\right)^{2}\right] /\left(2 R T_{b}\right)}=R T_{b} f_{1} .
\end{aligned}
$$

The energy flux may be represented in terms of the reduced distribution function as,

$$
\begin{aligned}
Q_{g}= & \alpha_{g} \frac{m}{2}\left[\int_{\mathbf{s} \cdot \mathbf{n}>\mathbf{0}} v\left(\mathbf{s}_{\mathbf{x y}} \cdot \mathbf{n}\right)\left(f_{1} v^{\prime 2}+f_{2}\right) v d v d \varphi\right. \\
& \left.+\int_{\mathbf{s} \cdot \mathbf{n} \leq \mathbf{0}} v\left(\mathbf{s}_{\mathbf{x y}} \cdot \mathbf{n}\right)\left(f_{1 \text { interface }} v^{\prime 2}+f_{2 \text { interface }}\right) v d v d \varphi\right],
\end{aligned}
$$

where $\mathbf{s}_{\mathbf{x y}}=\cos \phi \hat{i}+\sin \phi \hat{j}$

Here $s_{x y}$ is the direction of the velocity vector of gas molecules in the $x y$ plane. Once $f_{1}$ and $f_{2}$ are computed, bulk fluid properties may be calculated as,

$$
\begin{aligned}
& \text { Gas Density: } \rho=m \int_{0}^{\infty} \int_{0}^{2 \pi} f_{1} d \varphi v d v \\
& \text { Bulk } x \text { velocity: } u_{0}=\int_{0}^{\infty} \int_{0}^{2 \pi} v \cos \varphi f_{1} d \varphi v d v,
\end{aligned}
$$


Bulk $y$ velocity: $v_{0}=\int_{0}^{\infty} \int_{0}^{2 \pi} v \sin \varphi f_{1} d \varphi v d v$,

Temperature: $T=\frac{1}{3 R_{g}} \int_{0}^{\infty} \int_{0}^{2 \pi}\left[\left(\left(v \cos \varphi-u_{0}\right)^{2}\right.\right.$

$$
\left.\left.+\left(v \sin \varphi-v_{0}\right)^{2}\right) f_{1}+f_{2}\right] d \varphi v d v
$$

Scalar Pressure: $P=\rho R_{g} T$.

${ }^{1}$ D. G. Cahill, W. K. Ford, K. E. Goodson, G. D. Mahan, A. Majumdar, H. J. Maris, R. Merlin, and S. R. Phillpot, J. Appl. Phys. 93, 793 (2003).

${ }^{2}$ M. S. Dresselhaus, M. S. Lin, T. Koga, S. B. Cronin, O. Rabin, M. R. Black, and G. Dresselhaus, Semiconductors and Semimetals: Recent Trends in Thermoelectric Materials Research III (Academic, New York, 2001), Vol. 71, pp. 1-121.

${ }^{3}$ D. M. Rowe, Thermoelectrics Handbook: Macro to Nano (CRC, Boca Raton, 2005).

${ }^{4}$ I. Boukai, Y. Bunimovich, J. Tahir-Kheli, Yu. Jen-Kan, W. A. Goddard III, and J. R. Heath, Nature (London) 451, 168 (2007).

${ }^{5}$ A. I. Hochbaum, R. Chen, R. D. Delgado, W. Liang, E. C. Garnett, M. Najarian, A. Majumdar, and P. Yang, Nature (London) 451, 163 (2008).

${ }^{6}$ Y. Cui, Z. Zhong, D. Wang, W. U. Wang, and C. M. Lieber, Nano Lett. 3, 149 (2003).

${ }^{7}$ A. Javey, J. Guo, Q. Wang, M. Lundstrom, and H. Dai, Nature (London) 424, 654 (2003).

${ }^{8}$ J. Xu and T. S. Fisher, Int. J. Heat Mass Transfer 49, 1658 (2006).

${ }^{9}$ M. S. Ivanov and S. F. Gimelshein, Annu. Rev. Fluid Mech. 30, 469 (1998).

${ }^{10}$ J. C. Harley, Y. Huang, H. H. Bau, and J. N. Zemel, J. Fluid Mech. 284, 257 (1995).

${ }^{11}$ E. B. Arkilic, K. S. Breuer, and M. A. Schmidt, J. Fluid Mech. 437, 29 (2001).

${ }^{12}$ E. B. Arkilic, K. S. Breuer, and M. A. Schmidt, J. Microelectromech. Syst. 6, 167 (1997).

${ }^{13}$ X. Guo, C. Huang, A. Alexeenko, and J. Sullivan, J. Micromech. Microeng. 18, 025034, (2008).

${ }^{14}$ F. Pan, J. Kubby, E. Peeters, A. T. Tran, and S. Mukherjee, J. Micromech. Microeng. 8, 200 (1998).

${ }^{15}$ T. Veijola, J. Micromech. Microeng. 14, 1109 (2004).

${ }^{16}$ J. Lee, T. L. Wright, M. R. Abel, E. O. Sunden, A. Marchenkov, S. Graham, and W. P. King, J. Appl. Phys. 101, 014906, (2007).

${ }^{17}$ B. A. Nelson and W. P. King, Applied Scanning Probe Methods IV (Springer-Verlag, New York, 2006).

${ }^{18}$ W. P. King and K. E. Goodson, ASME J. Heat Transfer 124, 597 (2002).

${ }^{19}$ A. A. Alexeenko, D. A. Fedosov, S. F. Gimelshein, D. A. Levin, and R. J. Collins, J. Microelectromech. Syst. 15, 181 (2006).

${ }^{20}$ V. Bahadur, J. Xu, Y. Liu, and T. S. Fisher, ASME J. Heat Transfer 127, 164 (2005).

${ }^{21}$ R. Prasher, Nano Lett. 5, 2155 (2005).

${ }^{22}$ R. Prasher and P. Phelan, Proceedings of the ASME International Mechanical Engineering Congress and Exposition, Chicago, Illinois, 5-10 November 2006 (unpublished).

${ }^{23}$ D. Singh, T. S. Fisher, and J. Y. Murthy, Proceedings of the ASME InterPACK, Vancouver, British Columbia, Canada, 8-12 July 2007 (unpublished).

${ }^{24}$ M. Knudsen, Ann. Phys. 338, 16 (1910).

${ }^{25}$ G. Pham-Van-Diep, P. Keeley, E. P. Muntz, and D. P. Weaver, Rarefied
Gas Dynamics (Oxford University Press, Oxford, 1995), pp. 715-721.

${ }^{26}$ S. K. Loyalka, Phys. Fluids 12, 2301 (1969).

${ }^{27}$ Y.-L. Han, E. P. Muntz, A. Alexeenko, and M. Young, Nanoscale Microscale Thermophys. Eng. 11, 151 (2007).

${ }^{28}$ J. Y. Murthy, S. V. J. Narumanchi, J. A. Pascual-Gutierrez, T. Wang, C. Ni, and S. R. Mathur, Int. J. Multiscale Comp. Eng. 3, 5 (2005).

${ }^{29}$ G. Chen, Phys. Rev. B 57, 14958 (1998).

${ }^{30}$ R. Yang and G. Chen, Phys. Rev. B 69, 195316 (2004).

${ }^{31}$ C. Dames and G. Chen, J. Appl. Phys. 95, 682 (2004).

${ }^{32}$ J. Zou and A. Balandin, J. Appl. Phys. 89, 2932 (2001).

${ }^{33}$ N. Mingo, L. Yang, D. Li, and A. Majumdar, Nano Lett. 3, 1713 (2003).

${ }^{34}$ W. Zhang, N. Mingo, and T. S. Fisher, ASME J. Heat Transfer 129, 483 (2007).

${ }^{35}$ N. Mingo and L. Yang, Phys. Rev. B 68, 245406 (2003).

${ }^{36}$ J. R. Lukes and H. Zhong, ASME J. Heat Transfer 129, 705 (2007).

${ }^{37}$ S. Maruyama, Nanoscale Microscale Thermophys. Eng. 7, 41 (2003).

${ }^{38}$ A. J. H. McGaughey and M. Kaviany, Adv. Heat Transfer 39, 169 (2006).

${ }^{39}$ A. Henry and G. Chen, J. Comput. Theor. Nanoscience 5, 141 (2008).

${ }^{40}$ D. A. Broido, A. Ward, and N. Mingo, Phys. Rev. B 72, 014308 (2005).

${ }^{41}$ D. A. Broido, M. Malorny, G. Birner, N. Mingo, and D. A. Stewart, Appl. Phys. Lett. 91, 231922 (2007).

${ }^{42}$ G. A. Bird, Molecular Gas Dynamics and the Direct Simulation of Gas Flows (Oxford University Press, New York, 1994).

${ }^{43}$ L. Mieussens and H. Struchtrup, Phys. Fluids 16, 2797 (2004).

${ }^{44}$ L. Mieussens, J. Comput. Phys. 162, 429 (2000).

${ }^{45}$ L. Mieussens, Math. Models Meth. Appl. Sci. 10, 1121 (2000).

${ }^{46}$ P. Andries, B. Perthame, P. Le Tallec, and J. P. Perlat, Eur. J. Mech. B/Fluids 19, 813 (2000).

${ }^{47}$ J. A. Pascual-Gutiérrez, J. Y. Murthy, and R. Viskanta, J. Appl. Phys. 102, 034315 (2007).

${ }^{48}$ P. Andries, J. F. Bourgat, P. Le Tallec, and B. Perthame, Comput. Methods Appl. Mech. Eng. 191, 3369 (2002).

${ }^{49}$ W. J. Vincenti and C. H. Kruger, Introduction to Physical Gas Dynamics (Wiley, New York, 1965).

${ }^{50} \mathrm{C}$. Cercignani, Rarefied Gas Dynamics (Cambridge University Press, Cambridge, England, 2000).

${ }^{51}$ B. Shizgal, J. Comput. Phys. 41, 309 (1981).

${ }^{52}$ M. F. Modest, Radiative Heat Transfer (McGraw-Hill, New York, 1993).

${ }^{53} \mathrm{M}$. Gad-el-Hak, The MEMS Handbook, 1st ed. (CRC, Boca Raton, 2001).

${ }^{54}$ D. C. Wadsworth, Phys. Fluids A 5, 1831 (1993).

${ }^{55}$ M. A. Gallis, W. M. Trott, J. R. Torczynski, and D. J. Rader, Proceedings of the 59th Annual Meeting of the APS Division of Fluid Dynamics, Tampa Bay, Florida, 2006 (unpublished).

${ }^{56}$ F. O. Goodman, Prog. Surf. Sci. 5, 261 (1974).

${ }^{57}$ C. W. Muhlhausen, L. B. Williams, and J. C. Tully, J. Chem. Phys. 83, 2594 (1985).

${ }^{58}$ F. Sharipov, Eur. J. Mech. B/Fluids 22, 133 (2003).

${ }^{59}$ C. Cercignani and M. Lampis, Transp. Theory Stat. Phys. 1, 101 (1971).

${ }^{60}$ E. T. Schwartz and R. O. Pohl, Rev. Mod. Phys. 61, 605 (1989).

${ }^{61}$ P. K. Schelling, S. R. Phillpot, and P. Keblinski, Appl. Phys. Lett. 80, 2484 (2004).

${ }^{62}$ P. K. Schelling, S. R. Phillpot, and P. Keblinski, J. Appl. Phys. 95, 6082 (2004).

${ }^{63}$ A. Maali and B. Bhushan, Phys. Rev. E 78, 027302 (2008).

${ }^{64}$ R. Prasher, Phys. Rev. B 74, 165413 (2006).

${ }^{65}$ F. P. Incropera, D. P. Dewitt, T. L. Bergman, and A. S. Levine, Introduction to Heat Transfer, 5th ed. (Wiley, New York, 2006).

${ }^{66}$ M. N. Kogan, Rarefied Gas Dynamics (Plenum, New York, 1969).

${ }^{67}$ K.J. Daun, G. J. Smallwood, and F. Liu, ASME J. Heat Transfer 130, 121201 (2008).

${ }^{68}$ S. R. Mathur and J. Y. Murthy, ASME J. Heat Transfer 124, 1176 (2002). 